\title{
Direct anterior screw fixation for recent and remote odontoid fractures
}

\author{
Ronald I. APfelbaum, M.D., RuSSell R. LONSER, M.D., Robert Veres, M.D., \\ and Adrian Casey, M.D. \\ Department of Neurosurgery, University of Utah Health Sciences Center, Salt Lake City, Utah; \\ Department of Neurosurgery, National Institute of Traumatology, Budapest, Hungary; Department of \\ Surgical Neurology, The National Hospital for Neurology and Neurosurgery, Queen Square, London, \\ United Kingdom
}

\begin{abstract}
Object. The management of odontoid fractures remains controversial. Only direct anterior screw fixation provides immediate stabilization of the spine and may preserve normal $\mathrm{C} 1-2$ motion. To determine the indications, optimum timing, and results for direct anterior screw fixation of odontoid fractures, the authors reviewed the surgery-related outcome of patients who underwent this procedure at two institutions.

Methods. One hundred forty-seven consecutive patients ( 98 males and 49 females) who underwent direct anterior screw fixation for a recent fracture $(<6$ months postinjury [129 patients]) or remote $(\geq 18$ months postinjury [18 patients]) Type II (138 cases) or III (nine cases) odontoid fractures at the University of Utah (94 patients) and National Institute of Traumatology in Budapest, Hungary (53 patients) between 1986 and 1998 are included in this study (mean follow-up period 18.2 months). Data obtained from clinical examination, review of hospital charts, operative findings, and imaging studies were used to analyze the surgery-related results in these patients.

In patients with recent fractures there was an overall bone fusion rate of $88 \%$. The rate of anatomical bone fusion of recent fractures was significantly $(\mathrm{p} \leq 0.05)$ higher in fractures oriented in the horizontal and posterior oblique direction (as compared with anterior oblique), but this finding was independent $(\mathrm{p} \geq 0.05)$ of age, sex, number of screws placed (one or two), and the degree or the direction of odontoid displacement. In patients with remote fractures there was a significantly lower rate of bone fusion $(25 \%)$. Overall, complications related to hardware failure occurred in 14 patients $(10 \%)$ and unrelated to hardware in three patients $(2 \%)$. There was one death (1\%) related to surgery.

Conclusions. Direct anterior screw fixation is an effective and safe method for treating recent odontoid fractures $(<$ 6 months postinjury). It confers immediate stability, preserves $\mathrm{C} 1-2$ rotatory motion, and achieves a fusion rate that compares favorably with alternative treatment methods. In contradistinction, in patients with remote fractures $(\geq 18$ months postinjury) a significantly lower rate of fusion is found when using this technique, and these patients are believed to be poor candidates for this procedure.
\end{abstract}

KEY WordS - cervical spine injury - fracture • odontoid • screw fixation

Fractures of the odontoid process of the C-2 VB are common cervical spine injuries, accounting for 10 to $20 \%$ of all cervical spine fractures., $9,16,20$ These fractures most commonly occur at the junction of the odontoid process and the body of the C-2 (Type II) or into the body of C-2 (Type III) and result in atlantoaxial instability. Because of the instability associated with these lesions, patients are at significant risk for immediate or delayed catastrophic spinal cord compromise ${ }^{12}$ and achieving spinal stabilization is imperative.

Current methods for stabilization of odontoid fractures require rigid external immobilization or surgical fixation. External immobilization with a rigid orthosis can allow for the fracture to fuse without the need for surgical inter-

Abbreviations used in this paper: $\mathrm{AP}=$ anteroposterior; $\mathrm{CT}=$ computerized tomography; VB = vertebral body. vention, but it is limited because of higher nonunion rates and prolonged limitation of the patient's function. In surgical stabilization procedures surgeons have relied primarily on posterior atlantoaxial fusion in which they use a variety of bone and wire constructs. Whereas posterior fusion is associated with lower rates of nonunion, it eliminates normal $\mathrm{C} 1-2$ rotatory motion, which accounts for more than $50 \%$ of all cervical spine rotatory motion, and reduces cervical spine flexion-extension by $10 \% .^{42}$

To overcome the obstacles associated with commonly used odontoid fracture stabilization techniques, many centers have begun to use direct anterior screw fixation in the treatment of these fractures. Direct anterior screw fixation of odontoid fractures is an osteosynthetic technique that can provide immediate stability, promote bone healing, and may preserve normal $\mathrm{C} 1-2$ motion. Although this technique provides a potentially ideal method for the treat- 

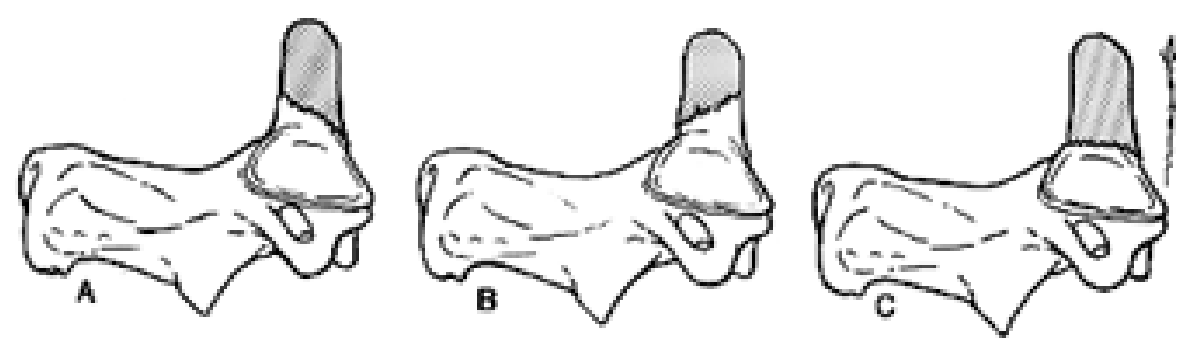

Fig. 1. Schematic illustration of the anterior oblique (A), posterior oblique (B), and horizontal (C) fracture classification. This classification scheme is based on the inferior direction of the slope of the fracture, as demonstrated on lateral radiographs.

ment of odontoid fractures, no published data exist regarding the indications, timing, and outcome in a large series of patients with these fractures who are treated with this method.

To best determine the indications, optimum timing, outcome, and the role of direct anterior screw fixation in the treatment of odontoid fractures, we examined a large series of patients in whom this technique was performed at two centers. Based on our findings, we then developed a treatment paradigm for managing these lesions.

\section{CLINICAL MATERIAL AND METHODS Patient Population}

Over a 13-year period (1986-1998) 147 consecutive patients underwent direct anterior screw fixation for Anderson and D'Alonzo ${ }^{4}$ Type II (138 patients) and shallow Type III (nine patients) odontoid fractures. These fractures were classified as recent $(<6$ months postinjury [129 cases]) or remote ( $\geq 18$ months postinjury [ 18 cases]). The procedures were performed at the University of Utah Health Sciences Center in Salt Lake City, Utah (94 patients) and the National Institute of Traumatology in Budapest, Hungary (53 patients). There were 98 male (67\%) and 49 female $(33 \%)$ patients who ranged in age from 15 to 92 years.

\section{Clinical Evaluation}

Patients underwent preoperative and serial postoperative (at approximately 6 weeks, 6 months, and yearly intervals thereafter) neurological examinations.

\section{Neuroimaging Evaluation}

All fractures were preoperatively assessed by evaluating the initial preoperative lateral and AP plain X-ray films and/or unenhanced CT scans of the odontoid. Serial postoperative AP and lateral flexion-extension plain x-ray films of the cervical spine were obtained concurrently at clinical visits to evaluate screw position, fracture alignment, and fusion status. Postoperative CT scanning was also used to augment plain x-ray film studies in some cases. Anatomical bone fusion was considered successful if there was trabeculation across the fracture site, the absence of movement on lateral flexion-extension radiographic studies, and anatomical alignment of the fracture fragment. Nonanatomical bone union was considered to have occurred if there was trabeculation across the fracture site, the absence of movement on flexion-extension films, and the presence of nonanatomical alignment of the odontoid fracture fragment. The presence of fibrous union was accepted if a visible fracture line was present and movement was absent on flexion-extension x-ray films.

To determine the effects of fracture orientation, the direction of odontoid displacement, and the degree of odontoid displacement on anatomical bone union, the radiographic characteristics of the fractures were recorded. Fracture type was determined by the criteria delineated previously by Anderson and D'Alonzo. ${ }^{4}$ Fracture orientation was classified according to the AP direction of the fracture line (anterior oblique, posterior oblique, or horizontal) (Fig. 1). The degree of displacement was determined by the percentage of displacement of the fractured odontoid fragment relative to the underlying body of C-2. The direction of odontoid displacement was classified as posterior, anterior, or neutral relative to the body of C-2.

\section{Operative Technique}

Click here to download video clip $88 \mathrm{MB}$ ].

The operative technique (see Video Clip) used in all

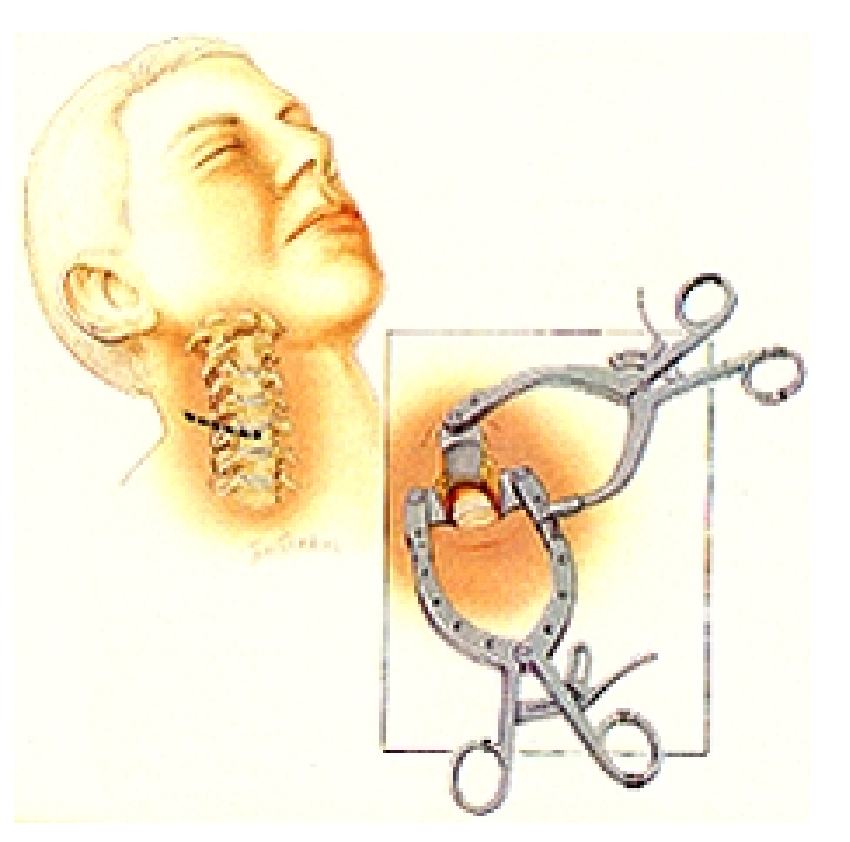

Fig. 2. Artist's drawing depicting the location of neck incision at the level of C-5. Inset: The position of the skin and soft-tissue retractors. 
cases at the University of Utah has been previously described by the senior author (R.I.A.). ${ }^{5}$ Briefly, the patient is placed supine on the operating table and positioned in such a manner that the odontoid fracture is best oriented so as to achieve optimum reduction. Halter, tong, or halo traction $(5-10 \mathrm{lbs})$ is used to hold the head immobile. The patient's mouth is kept open with a radiolucent jaw distractor. Fluoroscopic images are obtained in the AP and lateral planes.

The patient's neck is prepared and draped, and a unilateral horizontal incision is made at approximately the level C-5 (Fig. 2). The platysma is then elevated and divided, and the fascia of the sternocleidomastoid is sharply incised along its medial border. Blunt dissection is used to expose the anterior surface of the spinal column at the midcervical level by opening natural planes medial to the carotid artery sheath and lateral to the trachea and esophagus. The fascia of the musculus longus colli is incised in the midline, and the muscle is elevated from theVBs at the C5-6 level.

Sharp, large-toothed Caspar retractor blades are then inserted beneath the musculus longus colli bellies bilaterally and secured with a special lateral self-retaining retractor (Fig. 2 inset). This forms a stable base for the rostral retraction. Blunt dissection in the retropharyngeal space is used to open a tunnel in front of the VBs to the C-2 level.

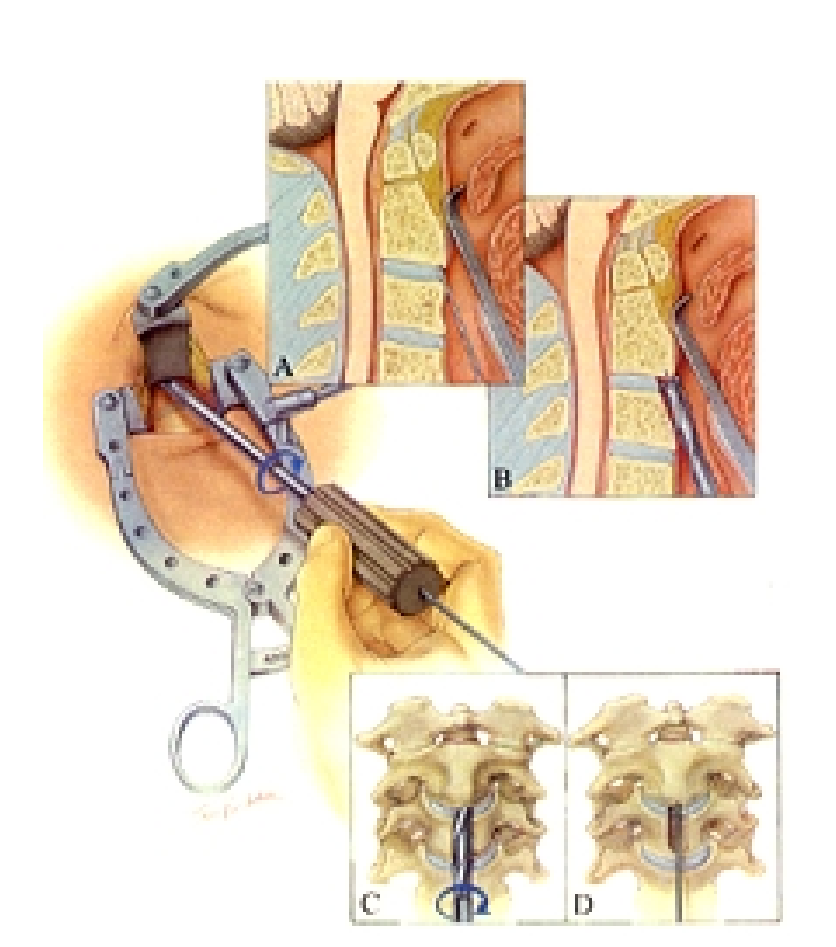

Fig. 3. Artist's drawing. Blunt dissection is used in the retropharyngeal space to open a tunnel in front of the VB to the C-2 level. A 2-mm K-wire is then manipulated while under biplanar fluoroscopic control 3 to $5 \mathrm{~mm}$ into the base of C-2. A: Sagittal view showing an appropriate-sized angled retractor elevating the retropharyngeal soft tissues anteriorly to expose the C-2 and C-3 VBs. The K-wire is in place. B: Once the guide wire (K-wire) is at the desired entry point in the C-2 body, a hollow 8-mm drill is placed over the wire. $\mathrm{C}$ and $\mathrm{D}$ : The drill is rotated by hand to create a shallow groove in the face of C-3 and the C2-3 disc and annulus without removing any of the $\mathrm{C}-2$ body.
An angled retractor of the appropriate size is then inserted into this space and coupled to the lateral retractors.

A K-wire is then inserted through the incision, up to the inferior edge of C-2, under fluoroscopic control and impacted into the inferior edge of C-2 (Fig. 3). If a single screw is placed, a midline entry site is chosen. A paramedian position, approximately 2 to $3 \mathrm{~mm}$ off the midline, is used if two screws are placed.

A hollow 8-mm drill is placed over the $\mathrm{K}$-wire and rotated by hand to create a shallow groove in the face of $\mathrm{C}$ 3 and the C2-3 disc and annulus to the inferior border of $\mathrm{C}-2$ without removing any of $\mathrm{C}-2$ (Fig. 3). The drill guide system is then placed over the K-wire (Fig. 4). A plastic impactor cover is placed over these, and the spikes of the outer guide tube are firmly set into C-3 under fluoroscopic guidance. The inner drill guide is then extended to contact the inferior edge of C-2. The alignment of the C-2 and C3 vertebrae relevant to the odontoid and $\mathrm{C}-1$ is controlled by the drill guide because it is firmly fixed to C-3 (Fig. 4). This allows for further extension of the neck if needed while correcting and maintaining optimum alignment.

Once the guide tubes are secured, the K-wire is removed and replaced with a drill bit, which engages the starter hole made by the $\mathrm{K}$-wire. A right-angle drive is used to clear the thoracic region, and a hole is then drilled while under careful biplane fluoroscopic control from the inferior anterior edge of C-2 through the body of C-2 and into the odontoid to its apex (Fig. 5). The drill is calibrated to allow accurate depth measurement. The drilled hole

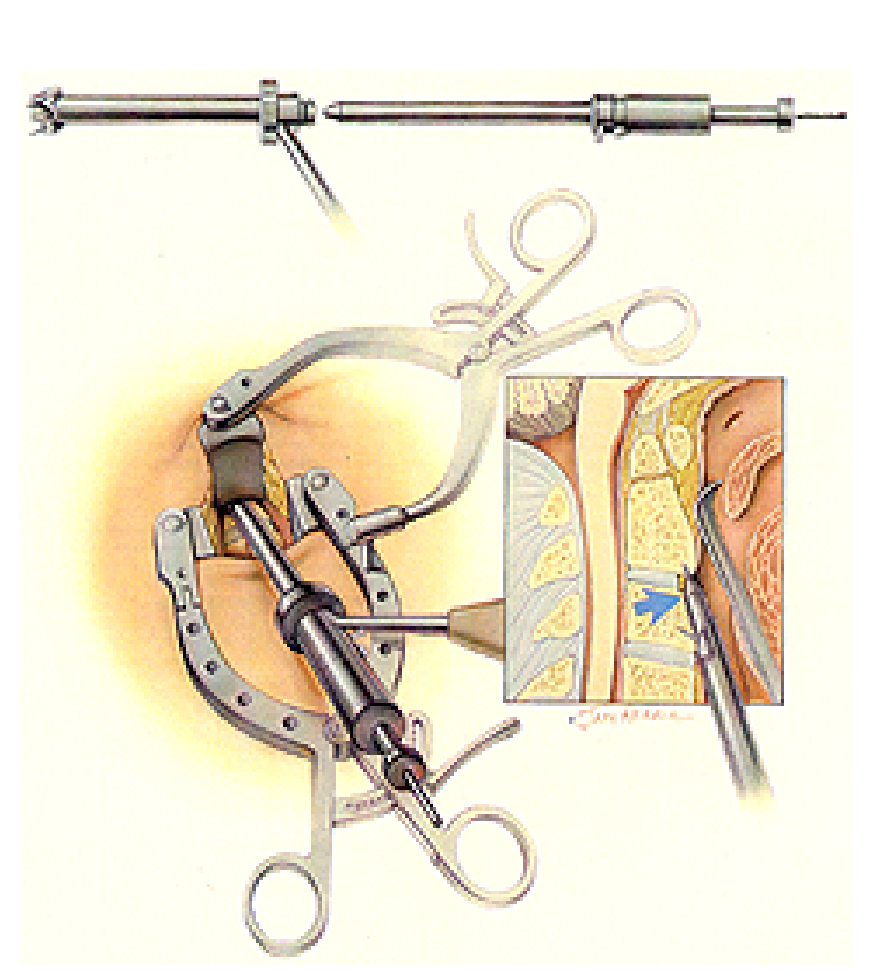

Fig. 4. Artist's illustrations. The inner and outer drill guide tubes are mated together and then placed over the K-wire. Inset: The guide system is manipulated into place and then secured by placing the outer drill guide spikes into C-3. The inner drill guide is then advanced in the previously created trough (arrow) to the inferior edge of $\mathrm{C}-2$. 


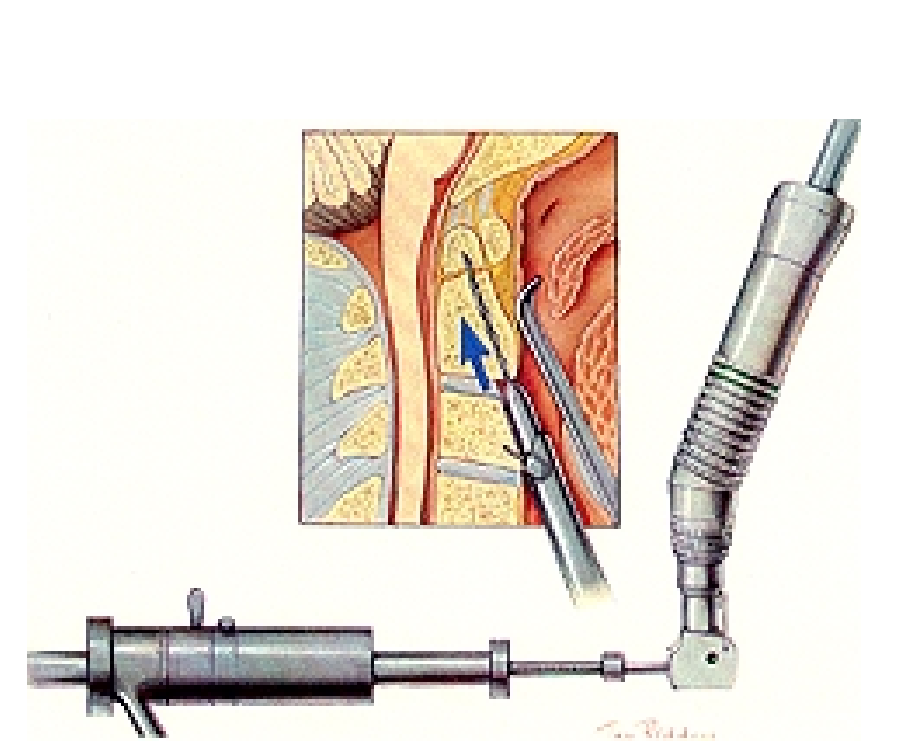

Fig. 5. Artist's drawing. Once the guide tubes are secured, the $\mathrm{K}$-wire is removed and replaced with a drill bit that is engaged in the starter hole (inset). The right-angle drill is then attached to the bit, and a hole is drilled under careful fluoroscopic guidance to the apex of the odontoid. The drill is calibrated to allow accurate depth measurement.

is then tapped (threaded) by removing the drill and the inner drill guide, replacing them with the tap that is manipulated by hand while monitoring its progress fluoroscopically (Fig. 6).

The screw, selected based on the measured depth, is placed through the outer guide tube and into the C-2 body through the drilled and tapped hole (Fig. 7). We use lag screws with a nonthreaded proximal shaft to allow the distal fragment to be pulled down to the body of C-2. The screw(s) is placed into the odontoid and tightened firmly, as progress is monitored fluoroscopically. The head of the screw is recessed into the C2-3 annulus/disc edge or into the inferior edge of $\mathrm{C}-2$, and the screw tip is fully engaged into the apical cortex of the odontoid. Extension by several millimeters beyond the tip is safe, as this will result in the screw tip being within the apical ligaments. The angle of placement is such that the neural elements are not jeopardized. In remote nonunited fractures, the screw is placed into the body of C-2 until just below the fracture site. The drill guide is then removed and special bisurfaced, angled curettes are used to freshen the fracture site and remove fibrous tissue (Fig. 7). This is performed by forcing the tip of the smaller curette through the weak anterior longitudinal ligament at the fracture site (as monitored fluoroscopically) and rotating the handle. It is then replaced with the second small curette angled in the opposite direction, which is manipulated similarly. Two larger curettes are then sequentially introduced and manipulated in the same manner. The screw head is then reengaged by the ball driver, which can be inserted at an angle of $\pm 15^{\circ}$ to the long axis of the screw, and tightened fully (Fig. 7).

Placement of a second screw is accomplished in a similar manner, using the K-wire for initial guidance, and an $8-\mathrm{mm}$ drill is used to cut a groove in the anterior surface of $\mathrm{C}-3$ and the $\mathrm{C} 2-3$ disc. As in cases in which a single screw is used, the surgeon places the drill guide, drills the hole, taps the hole, and places the screw. The second screw is either a lag screw or a fully threaded one.
R. I. Apfelbaum, et al.

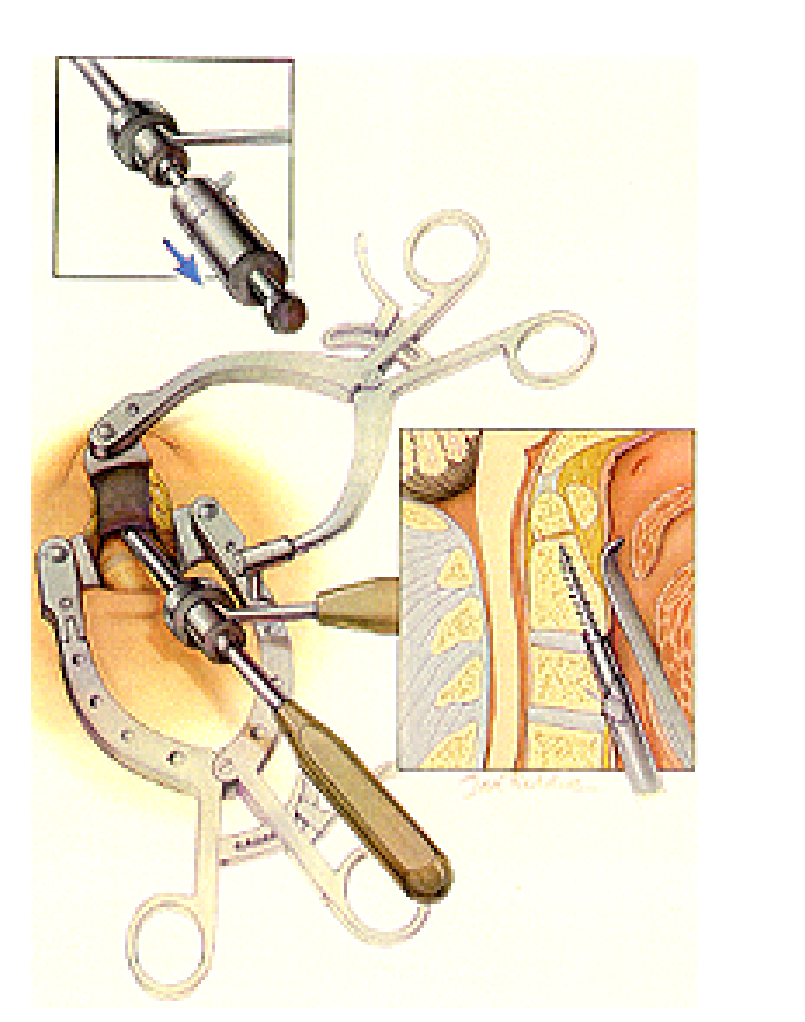

Fig. 6. Artist's illustrations. After an appropriate-sized hole is drilled to the apex of the odontoid, the inner guide tube is removed (upper inset) and a tap (lower inset) is used to cut threads into the pilot hole.

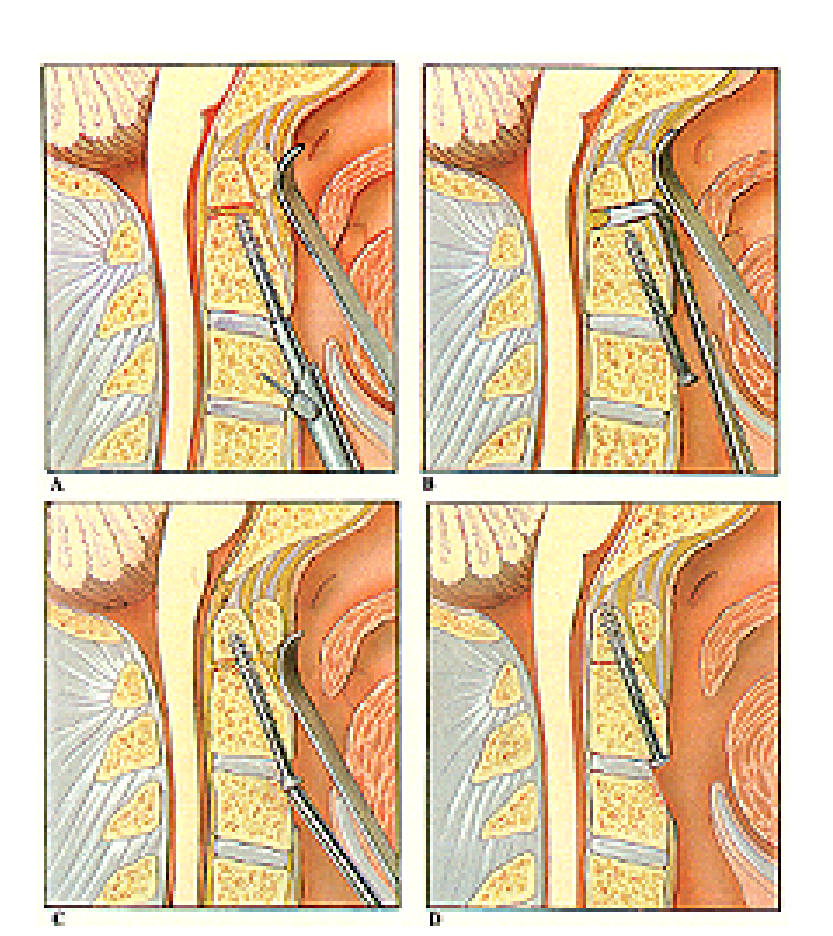

Fig. 7. Drawings illustrating the placement of screw(s) in the odontoid. A: The screw is inserted through guide tube and advanced through the threaded pilot hole. B: In remote fractures (those not treated until after 18 months postinjury), special bifaced curettes are used to freshen the fracture site. C: The screw is then advanced using a ball driver. D: Final screw position. 


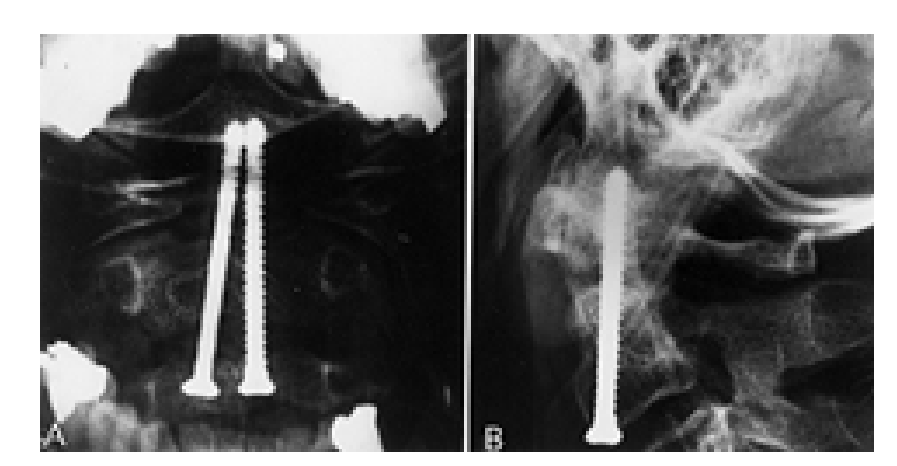

Fig. 8. Plain radiographs obtained in the $\mathrm{AP}(\mathrm{A})$ and lateral planes (B), revealing correct placement of two screws across the odontoid fracture site.

At this point, flexion-extension of the patient's neck under fluoroscopy is used to confirm spinal stability. The retractors are then removed, the wound checked for hemostasis, and closure completed in layers. We use interrupted absorbable sutures in the sternocleidomastoid muscle fascia, platysma muscles, and subcutaneous tissues, and we use sterile adhesive strips on the skin. Patients are not required to wear cervical collars postoperatively unless we observed radiographic evidence of osteopenia or the presence of associated cervical fractures.

The technique used at the National Institute of Traumatology in Budapest Hungary was similar, but it was undertaken without using the guide tube system anchored to C3 . This precluded using screw fixation of some posteriorly displaced odontoid fractures.

\section{Statistical Analysis}

Statistical significance was set at $\mathrm{p} \leq 0.05$. The chisquare test was used to analyze variables between groups.

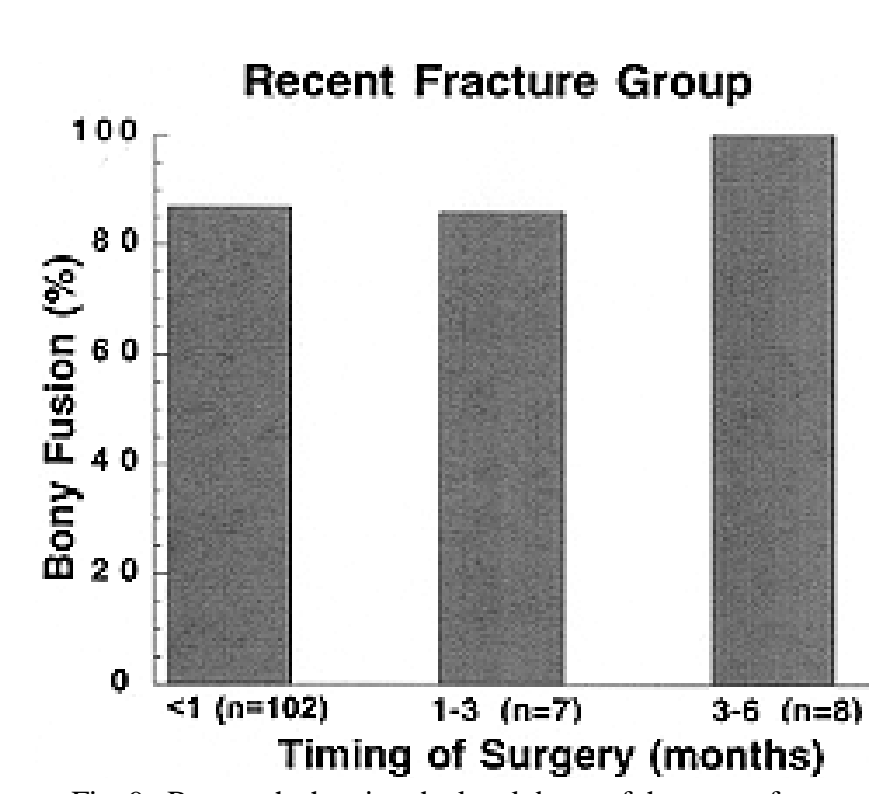

Fig. 9. Bar graph showing the breakdown of the recent fracture group. Similar fusion rates are demonstrated among those patients who underwent surgery on less then 1 month after injury, between 1 month and 3 months after injury, and between 3 months and 6 months after injury. Figures 2-8 are reproduced with permission from American Association of Neurological Surgeons.
TABLE 1

Characteristics obtained in 147 patients who underwent direct anterior screw fixation

\begin{tabular}{lcc}
\hline \hline \multicolumn{1}{c}{ Characteristic } & Recent Group & Remote Group \\
\hline sex (male/female) & $84: 45$ & $14: 4$ \\
mean age (yrs) & 52 & 41 \\
range of follow up (mos) & $3-60$ & $3-52$ \\
period of injury to op (range) & $1-180$ days & $18-48$ mos \\
\hline
\end{tabular}

\section{RESULTS}

\section{Patient Characteristics}

One hundred forty-seven patients underwent direct anterior screw fixation for recent $(<6$ months postinjury; [129 cases]) or remote ( $\leq 18$ or more months postinjury; [18 cases]), Type II ([138 cases]) or shallow Type III (nine cases) odontoid fractures at the University of Utah (94 patients) and the National Institute of Traumatology (53 patients). There were 98 male (67\%) and 49 female (33\%) patients included in this study (Table 1). Seven patients $(5 \%)$ were lost to follow up and seven patients $(5 \%$; all in patients with recent fractures) included in this study died (all within 30 days of surgery); thus 133 patients underwent follow-up examination. The overall mean age at surgery was 50.1 years (range 15-92 years), and overall mean follow-up period was 18.2 months (range 3-60 months). The most common mechanism of injury (46\%) was motor vehicle accident (Table 2). The preoperative clinical findings are listed in Table 3. One hundred seventeen patients $(80 \%)$ underwent placement of two screws at the time of surgery, and $30(20 \%)$ underwent placement of one screw at the time of surgery.

\section{Surgery-Related Outcome in Recent Fractures}

In 104 (80\%) of 129 patients with recent fractures two screws were placed, and in 25 patients $(20 \%)$ one screw was placed at the time of surgery. Immediate spinal stabilization was achieved in all patients as evidenced radiographically by successful fracture alignment and correction of abnormal translatory motion at $\mathrm{C} 1-2$. Successful positioning of the odontoid screw(s), as determined by postoperative neuroimaging, was obtained in 125 patients (97\%) (Fig. 8). There were four patients, all in the Hungarian series, in whom screw placement was shown to be

TABLE 2

Mechanism of injury

\begin{tabular}{lccc}
\hline \hline & \multicolumn{3}{c}{ No. of Patients (\%) } \\
\cline { 2 - 4 } \multicolumn{1}{c}{ Mechanism } & $\begin{array}{c}\text { All } \\
\text { Fractures }\end{array}$ & $\begin{array}{c}\text { Recent } \\
\text { Fractures }\end{array}$ & $\begin{array}{c}\text { Remote } \\
\text { Fractures }\end{array}$ \\
\hline motor vehicle accident & $68(46)$ & $58(45)$ & $10(56)$ \\
fall & $64(44)$ & $63(49)$ & $1(6)$ \\
blow to the head & $3(2)$ & $1(1)$ & $2(11)$ \\
diving injury & $2(1)$ & $2(2)$ & $0(0)$ \\
bicycle accident & $2(1)$ & $2(2)$ & $0(0)$ \\
unknown & $8(5)$ & $3(2)$ & $5(28)$ \\
total & 147 & $129(88)$ & $18(12)$ \\
\hline
\end{tabular}


R. I. Apfelbaum, et al.

TABLE 3

Preoperative neurological status

\begin{tabular}{lc}
\hline \hline \multicolumn{1}{c}{ Preop Finding } & All Patients (\%) \\
\hline no deficits or pain only & $133(90)$ \\
partial deficit & $12(9)$ \\
complete deficit (quadriplegia) & $2(1)$ \\
total & $147(100)$ \\
\hline
\end{tabular}

imprecise. All four of these patients underwent placement of two screws at the time of surgery. In each case, only one of the screws was found to be incorrectly placed. Two of these patients were placed in a halo orthosis, and satisfactory fusion was eventually demonstrated. The other two patients developed nonunions and underwent successful posterior C1-2 fusion several months later.

Five patients in this group were lost to follow up, and seven patients died (all within 30 days of the procedure) leaving 117 patients for whom follow-up data could be analyzed. Of these patients, 102 underwent surgery within 1 month of injury, seven between 1 month and 3 months of injury, and eight between 3 months and 6 months of injury. Of the 117 patients for whom follow-up data were available overall, successful anatomical bone fusion was seen in $99(85 \%)$, nonanatomical bone fusion in four (3\%), fibrous union in four (3\%), and nonunion in $10(9 \%)$ (Table 4). The fusion rates were similar among the patients in whom surgery was performed at less than 1 month posttrauma (87\%), 1 to 3 months posttrauma (86\%), and 3 to 6 months posttrauma (100\%) (Fig. 9). All patients were neurologically stable after surgery and remained so as of last follow-up visit. The four patients in whom nonanatomical bone union was observed were without pain or neurological sequelae, and they have not required additional treatment. The 10 patients with fracture nonunion were treated either nonsurgically in hard cervical collars (six patients) or surgically (four patients). The six patients with nonunion who are undergoing cervical collar therapy are currently without pain, and their fusion status is pending. The four patients treated surgically underwent either posterior fusion to correct for an imprecisely placed screw (two cases) or reoperation with direct anterior screw replacement (two cases). In all four of these patients successful fusion was eventally demonstrated.

Statistical Analysis. Chi-square analysis of the effect of patient sex, age, fracture type, fracture orientation, num-

TABLE 4

Postoperative fusion status in the recent fracture group

\begin{tabular}{lccc}
\hline \hline & \multicolumn{3}{c}{ No. of Patients (\%) } \\
\cline { 2 - 4 } \multicolumn{1}{c}{ Fusion Status } & $\begin{array}{c}\text { Overall } \\
(117 \text { cases }) *\end{array}$ & $\begin{array}{c}\text { Utah } \\
(64 \text { cases })\end{array}$ & $\begin{array}{c}\text { Budapest } \\
\text { (53 cases) }\end{array}$ \\
\hline bone union & $99(85)$ & $55(86)$ & $44(83)$ \\
nonanatomical bone union & $4(3)$ & $2(3)$ & $2(4)$ \\
fibrous union & $4(3)$ & $4(6)$ & $0(0)$ \\
nonunion & $10(9)$ & $3(5)$ & $7(13)$ \\
total & $117(100)$ & 64 & 53 \\
\hline
\end{tabular}

TABLE 5

Chi-square analysis of variables affecting successful fusion in patients who underwent surgery before 6 months postinjury*

\begin{tabular}{lccc}
\hline \hline \multicolumn{1}{c}{ Variable } & $\begin{array}{c}\text { No. of } \\
\text { Cases }\end{array}$ & $\begin{array}{c}\mathrm{X}^{2} \\
\text { Value }\end{array}$ & p Value \\
\hline patient age & 117 & 3.4 & $\mathrm{NS}$ \\
patient sex & 117 & 1.8 & $\mathrm{NS}$ \\
no. of screws & 117 & 0.08 & $\mathrm{NS}$ \\
fracture orientation & 16 & 20.2 & 0.0001 \\
$\quad$ anterior oblique & 34 & 0.01 & $\mathrm{NS}$ \\
$\quad$ posterior oblique & 49 & 0.06 & $\mathrm{NS}$ \\
$\quad$ horizontal & 115 & 9.6 & $\mathrm{NS}$ \\
fracture displacement direction & 115 & 3.1 & $\mathrm{NS}$ \\
degree of fracture displacement & & &
\end{tabular}

$* \mathrm{NS}=$ not significant.

ber of screws placed (one or two), and degree of odontoid displacement revealed that anatomical bone fusion was significantly $(\mathrm{p} \leq 0.05)$ affected by fracture orientation only (Table 5). Fractures oriented in the anterior oblique orientation were significantly $(\mathrm{p} \leq 0.05)$ more likely to result in nonanatomical union, fibrous union, or nonunion than posterior oblique and horizontal oriented fractures.

Complications. Postoperative hardware-related complications occurred in $10(9 \%)$ of the 117 patients with recent fracture for whom follow-up data were available (Table 6). The most common hardware-related failure was screw pullout of the body of C-2 prior to development of fusion. This complication occurred in five patients, all of whom had associated fractures extending into the body of C-2, which were recognized preoperatively, but were believed still to afford an acceptable substrate for screw fixation. Successful fusion was achieved without neurological sequelae in two of these patients who were managed with a halo orthosis and a hard cervical collar, respectively. The other two patients with C-2 screw pullout were treated surgically. One patient underwent posterior $\mathrm{C} 1-2$ stabilization, and the other underwent direct anterior screw replacement. In both cases successful fusion was eventually demonstrated. Screw backout was the second most common hardware-related complication and was seen in four patients. In each case, the screws only partially engaged the apical odontoid cortex. One patient underwent posterior $\mathrm{C} 1-2$ fusion and one direct anterior screw replace-

TABLE 6

Hardware-related complications

\begin{tabular}{lccc}
\hline \hline & \multicolumn{3}{c}{ No. of Complications $(\%)^{*}$} \\
\cline { 2 - 4 } \multicolumn{1}{c}{ Complication } & $\begin{array}{c}\text { Both } \\
\text { Groups }\end{array}$ & $\begin{array}{c}\text { Recent } \\
\text { Group }\end{array}$ & $\begin{array}{c}\text { Remote } \\
\text { Group }\end{array}$ \\
\hline screw pullout of C-2 VB & $5(4)$ & $5(4)$ & $0(0)$ \\
screw pullout of the odontoid & $1(1)$ & $0(0)$ & $1(6)$ \\
screw backout & $4(3)$ & $4(3)$ & $0(0)$ \\
screw fracture & $3(2)$ & $0(0)$ & $3(19)$ \\
C-2 subluxation & $1(1)$ & $1(1)$ & $0(0)$ \\
total & $14(10)$ & $10(9)$ & $4(25)$ \\
\hline
\end{tabular}

* Percentages expressed as the ratio of the number of complications and number of patients for whom follow-up data were available in each group. 
ment, and fusion was subsequently observed in both cases. In the third patient fusion was achieved after treatment in a halo orthosis. The fourth patient from the Hungarian series became quadriplegic after the distal fracture fragment became dislocated subsequent to the screw backing out 3 weeks after surgery. This patient was placed in skeletal traction but died of respiratory causes prior to operative intervention. In addition to hardware-related complications, two (2\%) patients suffered superficial wound infections that were treated successfully with a course of oral antibiotics. The seven patients $(6 \%)$ who died within 30 days of the procedures died of nonsurgeryrelated causes, except for the one patient (1\%) with respiratory-related causes.

\section{Surgery-Related Outcome in Remote Fractures}

All patients in this group underwent direct anterior screw fixation 18 or more months after injury (Table 1). In 13 patients $(72 \%)$ two screws were placed and in five patients $(28 \%)$ one screw was placed at the time of surgery. Immediate stabilization was achieved in all patients, as evidenced radiographically by successful fracture realignment and correction of abnormal translatory motion at C1-2. Successful positioning of the odontoid screw(s), as determined by postoperative neuroimaging, was obtained in 16 patients $(89 \%)$. Imprecise placement of odontoid screws occurred in two patients $(11 \%)$, both in the Utah series. In one patient, the screw was too long and extended $2 \mathrm{~mm}$ beyond the tip of the odontoid. In the second patient two screws that were placed far too lateral and not entirely within the body of the odontoid. Both patients were returned to the operating room 2 days later and underwent successful replacement of the screws.

Follow-up data were available for 16 patients (two did not attend follow up). Overall, successful bone fusion was obtained in four patients $(25 \%)$, fibrous union in seven (44\%), and nonunion in five $(31 \%)$ (Table 7$)$. As of last follow up all patients remained stable. In the five patients with nonunion there was clear evidence of motion with loosening (two cases) or fracture (three cases) of the screws. In four of these patients $\mathrm{C} 1-2$ transarticular screws were subsequently placed and successful fusion was achieved. The fifth patient in this group has refused further treatment.

Complications. Hardware-related problems occurred in four patients (25\%; Table 6). The most common problem was screw fracture, which occurred in three patients in whom fusion did not occur. These patients underwent suc-

TABLE 7

Postoperative fusion status in patients who underwent surgery after 18 months postinjury*

\begin{tabular}{lc}
\hline \multicolumn{1}{c}{ Fusion Status } & No. of Patients $(\%)$ \\
\hline bony union & $4(25)$ \\
nonanatomical bone union & $0(0)$ \\
fibrous union & $7(44)$ \\
nonunion & $5(31)$ \\
total & $16(100)$ \\
\hline
\end{tabular}

* Two patients did not attend follow up after surgery in this group, leaving 16 patients for whom follow-up results could be evaluated. cessful C1-2 posterior fusion. Screw backout occurred in one patient, who also underwent successful $\mathrm{C} 1-2$ posterior fusion. In addition to hardware-related complications, one patient (6\%) suffered a small esophageal leak at C5-6, as evidenced after the patient ingested contrast medium. This patient was treated successfully for 3 days by denial of oral intake and placement of a nasogastric tube.

\section{DISCUSSION}

\section{Treatment of Odontoid Fractures}

Fractures of the odontoid process were classified into three types based on anatomical location by Anderson and D'Alonzo in $1974 .{ }^{4}$ Their classification scheme has proven useful in predicting atlantoaxial stability and evaluating various treatment modalities. Very rare, Type I fractures occur at the tip of the dens. They have in the past generally been considered stable, but this has been questioned more recently by Scott, et al. ${ }^{37}$ None of the patients in this study had sustained a Type I fracture. Type II fractures are the most common type of odontoid process fracture. They occur through the base or waist of the dens and are inherently unstable, they require either external immobilization or surgical treatment. Type III fractures occur through the body of C-2 and can be unstable; particularly if the fracture line is "shallow" in location or the distal fracture fragment is displaced. Subsequently, these fractures also require external immobilization or, in some cases, surgical treatment.

Because various methods of treatment exist for Types II and III odontoid fractures, considerable controversy exists regarding the best method for stabilizing these fractures. Initial treatment has relied primarily on immobilization by using a rigid orthosis such as a halo vest or a Minerva jacket. Reported success with such immobilization has varied widely from 7 to $100 \% .^{29,30}$ In most series, however, the authors report rates of successful fusion from 37 to $75 \%$. 2,6,7,11,16,17,20,22,24,34-36,41 The relatively high failure rate associated with external immobilization of odontoid fractures, coupled with the direct and indirect costs of this method of treatment, have led several authors to attempt to identify patients who would most likely be successful surgical candidates for fracture fixation. The results of such studies have suggested that the degree of dens dislocation $(>4-6 \mathrm{~mm}),{ }^{6,22}$ increasing age $(>40-65$ years $),{ }^{6,17}$ posterior odontoid subluxation, ${ }^{17}$ and comminution of the base of the dens ${ }^{23}$ would predict lower rates of fracture union. As a result, patients fitting into one or more of these categories or who refuse external immobilization are considered as surgical candidates.

Surgical stabilization for odontoid fractures has relied primarily on various methods of $\mathrm{C} 1-2$ posterior fusion. Such posterior stabilization techniques are associated with rates of fusion similar to those seen with direct anterior screw fixation ${ }^{3,10,15,39}$ but, when successful, result in the loss of normal $\mathrm{C} 1-2$ rotatory motion, which contributes to $50 \%$ of the normal rotatory excursion of the head and neck, and reduction of normal cervical flexion and extension by $10 \% .^{42}$ Moreover, to maximize fusion rates when these constructs are used many authors have advocated postoperative halo vest immobilization, which further limits patient function significantly. ${ }^{10,15}$ 
To overcome the limitations associated with either conservative or posterior surgical methods for odontoid fracture stabilization, surgeons at many centers have begun to use direct anterior screw fixation to treat Type II and some Type III fractures. Direct anterior screw fixation is an osteosynthetic technique that can provide immediate spinal stabilization. The results of clinical studies have shown that anterior screw fixation can preserve normal C1-2 rotatory motion. ${ }^{21,31}$ Montesano, et al. ${ }^{31}$ have reported that in $83 \%$ of their patients seen in follow up, full range of motion was maintained after anterior screw fixation of odontoid fractures.

Direct anterior screw fixation was originally advocated by Nakanishi ${ }^{32}$ in 1980 and later by Bohler ${ }^{8}$ who, in 1982, independently reported his experience dating back to 1968 . Despite sporadic reports from a number of surgeons, ${ }^{1,5,26-28,31,33}$ it was not until advances in the quality of intraoperative fluoroscopy and improvements in surgical instrumentation were made that this procedure gained wider acceptance.

\section{Operative Outcome Analysis}

Timing of Surgery. The timing of surgery for odontoid fractures is critical. Although there was no difference in fusion rates among subgroups of the recent fracture group (within 6 months of injury) who underwent surgery at less than 1 month, between 1 month and 3 months, and between 3 months and 6 months (usually the later two subgroups were treated surgically after failure of external immobilization treatment) (Fig. 9), there was a marked decrease in the rate of bone union in patients in whom surgery was performed after 18 months. Overall, in patients who underwent direct screw fixation within 6 months of injury an anatomic fusion rate of $88 \%$ was demonstrated, whereas in patients who underwent this procedure after 18 months postinjury (remote group), an anatomic fusion rate of $25 \%$ was found, despite the fact that the patients in the remote fracture group were younger (mean age 52 compared with 41 years, respectively) and presumably had healthier, stronger bone. Because there were no patients in this study in whom surgery was performed between 6 months and 18 months after injury, we are not able to draw conclusions as to the expected fusion rates for patients surgically treated during this time period.

The high rate of nonunion in the remote fracture group is likely related to changes that occur at the fracture site. In these patients, we typically found radiographic evidence of progressive sclerotic changes at the fracture edges. Sclerotic changes result in a poor environment for fracture healing, and indeed the results in this group bear this out despite our attempts to "freshen" the fracture site with curettage. Subsequently, it would seem best to treat odontoid fractures as early as is feasible to maximize the potential for bone union.

Factors Affecting Fusion. We evaluated the effects of age, sex, fracture orientation, direction of odontoid displacement, and degree of odontoid displacement on the rate of anatomical fusion in recent odontoid fractures (Table 5). The only variable that exerted a significant effect on anatomical bone union was the orientation of the odontoid fracture. In patients with anterior oblique fracture ori- entations (16 cases) a significantly ( $\mathrm{p} \leq 0.05)$ lower rate of anatomical bone fusion was found when compared with patients in whom posterior oblique or horizontal fracture orientations were demonstrated. A $50 \%$ bone union rate, a nonanatomic union rate of $25 \%$, and nonunion rate of $25 \%$ were found in patients with anterior oblique fractures. In the four cases of nonanatomical fusion no neurological sequelae were observed, and the patients have not required additional treatment. The increased rate of nonanatomical fusion and nonunion in these patients is probably due to shearing forces created by loading at the fracture site. The screw traversing the oblique fracture line cannot resist these forces well and also may tend to pull the distal fragment anteriorly and inferiorly, as postulated by Aebi, et al., ${ }^{1}$ and Dickman, et al. ${ }^{14}$

That there is no correlation with degree and direction of odontoid displacement reveals that these are grossly unstable fractures that can displace and relocate easily. Thus, any plain x-ray film study is simply a "snapshot in time," reflecting the current situation and frequently not the worst-case scenario. As such, a minimally displaced odontoid can be shown to be significantly malaligned on a subsequent radiograph. Indeed, we have observed fluoroscopically odontoid movement from $8 \mathrm{~mm}$ of anterior displacement to $8 \mathrm{~mm}$ of posterior displacement with each respiratory cycle in a patient in halo immobilization.

Number of Screws. Although the results of biomechanical studies have shown no increased strength with regards to bending movements between one- and two-screw constructs, a second screw may offer the theoretical advantage of preventing rotation of the odontoid relative to the body of C-2. Because of this potential advantage, we used two screws whenever there was an adequate dimension for the odontoid to accept two.

Overall, two screws were used in 117 (80\%) of 147 cases and one screw in 30 cases $(20 \%)$. There was no significant difference in overall rates of successful fusion between the groups (one- compared with two-screw constructs). This is consistent with a recent study reported by Jenkins, et al., ${ }^{26}$ in which the authors showed that there was no clinical advantage to using a two-screw construct.

Complications. Overall, complications related to hardware failure occurred in 14 patients $(10 \%)$ and unrelated to hardware in three patients $(2 \%)$. There was one death (1\%) related to surgery (in the recent group surgically treated in fewer than 6 months postinjury). The complication rate in this and other smaller series $1,8,9,18-21,25,26,28,31-33,40$ in which direct screw fixation for odontoid fractures was used is similar to that associated with posterior fixation techniques. ${ }^{3,10,15,38}$ The most common hardware-related failure was screw pullout of the body of C-2 (five cases). Each of these cases was in a patient with a fracture into the body of C-2. Although these fractures were recognized prior to surgery on radiographic studies, we believed (erroneously) that the body would still afford an acceptable substrate for fusion. Based on our current results, we no longer perform this procedure in patients in whom there is evidence of comminuted C-2 body fractures. The second most common hardware-related complication was screw backout, which occurred in four patients. In every case of screw backout, postoperative radiography revealed that the screw(s) did not completely cross the apical cortex. 
This finding emphasizes the need to cross the apical cortex completely with the screw threads so that adequate purchase of the screw occurs in the distal fragments.

Analysis of data obtained in the two fracture groups (recent and remote) revealed that in patients surgically treated after 18 months postinjury there was a significantly higher rate of hardware-related complications (25\%) when compared with those in the recent fracture group $(9 \%)$. This was not unexpected because all hardware is placed for fracture immobilization and ultimately fails if bone healing does not occur.

Imprecise placement of screw(s) at the time of surgery occurred in six patients. We found that hardware misplacement has become rarer as the intraoperative fluoroscopic capabilities have improved and biplanar fluoroscopy has been adopted.

\section{Treatment Paradigm}

Based on our results and the existing data, we have developed a treatment paradigm for recent $(<6$ months postinjury) odontoid fractures. The initial evaluation of odontoid fractures should begin with careful clinical evaluation and examination of the cervical spine plain x-ray films. Clinical or radiographic evidence of odontoid fracture should prompt the clinician to perform a serial thincut CT scanning through the skull base to the top of C-3. Reconstructed CT scans will provide the surgeon with an excellent idea of the fracture type, orientation, and the degree of dens displacement. Concerns about the integrity of the transverse ligament can be addressed by performing magnetic resonance imaging studies. ${ }^{13}$

Type I fractures may be treated with external immobilization, but need to be carefully assessed with regard to spinal stability. ${ }^{37}$ We believe that all recent Type II fractures with an intact transverse ligament should be treated with direct anterior screw fixation. Despite having a lower rate of anatomical bone union in this series, anterior oblique-oriented fractures still have a bone stabilization rate of $75 \%$ (50\% anatomic bone union and $25 \%$ nonanatomical union), which compares favorably with results obtained using most external immobilization modalities. To avoid nonanatomical union with this fracture orientation type, we would consider placing these patients in a hard cervical collar postoperatively until evidence of fibrous or bone union is present. In addition, fixation of anterior oblique fractures in a 1- to 2-mm posteriorly displaced position may anticipate anterior movement of the odontoid during the healing process and reduce nonanatomical outcomes. Because of the high rate of bone fusion found in Type III fractures treated with cervical immobilization, we still manage most of these fractures with external stabilization. Patients who refuse external immobilization or who have displaced fractures are considered as candidates for direct anterior screw fixation if their fracture is high in the body of C-2 and if it is not associated with comminution of the body of $\mathrm{C}-2$.

Because remote ( $>6$ months postinjury) odontoid fractures (Types II and III) have a significantly higher rate of nonunion, we recommend treating these lesions with posterior C1-2 fixation and fusion. We no longer offer direct anterior screw fixation except in a select subgroup of patients who have relatively large odontoid processes, which are not ankylosed to either the anterior arch of C-1 or the clivus, and who have a small gap $(<2 \mathrm{~mm})$ between the odontoid and the body of C-2. Such patients remain potential candidates for direct screw fixation, but they are cautioned that there is a lower incidence of successful fusion than in patients with recent fractures and that fusion may ultimately fail to occur, requiring posterior fusion.

\section{CONCLUSIONS}

Direct anterior screw fixation is an easily learned, costeffective, and relatively straightforward technique for treating odontoid fractures. It confers immediate stability and provides an optimum anatomical environment for bone healing. Successful osteosynthesis in $85 \%$ of cases and successful stabilization in $91 \%$ of cases were achieved in patients in whom this technique was used to treat fractures less than 6 months postinjury. In contradistinction in patients with fractures discovered 18 or more months after injury a significantly lower rate of fusion was found when using this technique.

\section{Disclosure Statement}

Dr. Apfelbaum serves as a consultant for Aesculap Inc. (South San Francisco, CA), whose instruments and screws were used to perform this operation.

\section{References}

1. Aebi M, Etter C, Coscia M: Fractures of the odontoid process. Treatment with anterior screw fixation. Spine 14:1065-1070, 1989

2. Althoff B: Fracture of the odontoid process. An experimental and clinical study. Acta Orthop Scand Suppl 177:1-95, 1979

3. Anderson LD: Fractures of the odontoid process of the axis, in Cervical Spine Research Society (ed): The Cervical Spine. Philadelphia: JB Lippincott, 1983, pp 206-223

4. Anderson LD, D'Alonzo RT: Fractures of the odontoid process of the axis. J Bone Joint Surg (Am) 56:1663-1674, 1974

5. Apfelbaum RI: Anterior screw fixation for odontoid fractures, in Rengachary SS, Wilkins RH (eds): Neurosurgical Operative Atlas, ed 3. Baltimore: American Association of Neurological Surgeons, 1992, Vol 2, pp 189-199

6. Apuzzo MLJ, Heiden JS, Weiss MH, et al: Acute fractures of the odontoid process. An analysis of 45 cases. J Neurosurg 48: 85-91, 1978

7. Blockey NJ, Purser DW: Fractures of the odontoid process of the axis. J Bone Joint Surg (Br) 38:794-817, 1956

8. Bohler J: Anterior stabilization for acute fractures and nonunions of the dens. J Bone Joint Surg (Am) 64:18-27, 1982

9. Borne GM, Bedou GL, Pinaudeau M, et al: Odontoid process fracture osteosynthesis with a direct screw fixation technique in nine consecutive cases. J Neurosurg 68:223-226, 1988

10. Brooks AL, Jenkins EB: Atlanto-axial arthrodesis by the wedge compression method. J Bone Joint Surg (Am) 60:279-284, 1978

11. Clark CR, White AA III: Fractures of the dens. A multicenter study. J Bone Joint Surg (Am) 67:1340-1348, 1985

12. Crockard HA, Heilman AE, Stevens JM: Progressive myelopathy secondary to odontoid fractures: clinical, radiological, and surgical features. J Neurosurg 78:579-586, 1993

13. Dickman CA, Mamourian A, Sonntag VKH, et al: Magnetic resonance imaging of the transverse atlantal ligament for the evaluation of atlantoaxial instability. J Neurosurg 75:221-227, 1991 
14. Dickman CA, Sonntag VKH, Marcotte PJ: Techniques of screw fixation of the cervical spine. BNI Q 8:9-26, 1992

15. Dickman CA, Sonntag VKH, Papadopoulos SM, et al: The interspinous method of posterior atlantoaxial arthrodesis. J Neurosurg 74:190-198, 1991

16. Dickson H, Engel S, Blum P, et al: Odontoid fractures, systemic disease and conservative care. Aust NZ J Surg 54:243-247, 1984

17. Dunn ME, Seljeskog EL: Experience in the management of odontoid process injuries: an analysis of 128 cases. Neurosurgery 18:306-310, 1986

18. Esses SI, Bednar DA: Screw fixation of odontoid fractures and nonunions. Spine 16 (Suppl 10):S484-S485, 1991

19. Etter C, Coscia M, Jaberg H, et al: Direct anterior screw fixation of dens fractures with a cannulated screw system. Spine 16 (Suppl 3):S25-S32, 1991

20. Fujii E, Kobayashi K, Hirabayashi K: Treatment in fractures of the odontoid process. Spine 13:604-609, 1988

21. Geisler FH, Cheng C, Poka A, et al: Anterior screw fixation of posteriorly displaced type II odontoid fractures. Neurosurgery 25:30-38, 1989

22. Hadley MN, Browner C, Sonntag VKH: Axis fractures: a comprehensive review of management and treatment in 107 cases. Neurosurgery 17:281-290, 1985

23. Hadley MN, Browner CM, Liu SS, et al: New subtype of acute odontoid fractures (type IIA). Neurosurgery 22:67-71, 1988

24. Hentzer L, Schalimtzek M: Fractures and subluxations of the atlas and axis. A follow-up study of 20 patients. Acta Orthop Scand 42:251-258, 1971

25. Jeanneret B, Vernet $\mathrm{O}$, Frei $\mathrm{S}$, et al: Atlantoaxial mobility after screw fixation of the odontoid: a computed tomographic study. J Spinal Disord 4:203-211, 1991

26. Jenkins JD, Coric D, Branch CL Jr: A clinical comparison of one- and two-screw odontoid fixation. J Neurosurg 89: 366-370, 1998

27. Knoringer P: Internal fixation of dens fractures by doublethreaded screws. Orthop Traumatol 1:231-245, 1992

28. Lesoin F, Autricque A, Franz K, et al: Transcervical approach and screw fixation for upper cervical spine pathology. Surg Neurol 27:459-465, 1987

29. Lind B, Nordwall A, Sihlbom H: Odontoid fractures treated with halo-vest. Spine 12:173-177, 1987

30. Maiman DJ, Larson SJ: Management of odontoid fractures. Neurosurgery 11:471-476, 1982
31. Montesano PX, Anderson PA, Schlehr F, et al: Odontoid fractures treated by odontoid anterior screw fixation. Spine 16 (Suppl 3):S33-S37, 1991

32. Nakanishi T: Internal fixation of the odontoid fracture. Cent Jpn J Orthop Traumatic Surg 23:399-406, 1980

33. Pentelenyi T, Szarvas I, Brodrogi L: Screw fixation of odontoid fractures: preliminary report. Injury 19:139-142, 1988

34. Pepin JW, Bourne RB, Hawkins RJ: Odontoid fractures, with special reference to the elderly patient. Clin Orthop (193): 178-183, 1985

35. Ryan MD, Taylor TKF: Odontoid fractures. A rational approach to treatment. J Bone Joint Surg (Br) 64:416-421, 1982

36. Schatzker J, Rorabeck CH, Waddell JP: Fractures of the dens (odontoid process). An analysis of thirty-seven cases. J Bone Joint Surg (Br) 53:392-405, 1971

37. Scott EW, Haid RW Jr, Peace D: Type I fractures of the odontoid process: implications for atlantooccipital instability. Case report. J Neurosurg 72:488-492, 1990

38. Song GS, Theodore N, Dickman CA, et al: Unilateral posterior atlanto-axial transarticular screw fixation. J Neurosurg 87: $851-855,1997$

39. Stillerman CB, Wilson JA: Atlanto-axial stabilization with posterior transarticular screw fixation: technical description of 22 cases. Neurosurgery 32:948-955, 1993

40. Subach BR, Morone MA, Haid RW Jr, et al: Management of acute odontoid fractures with single-screw anterior fixation. Neurosurgery 45:812-820, 1999

41. Wang GJ, Mabie KN, Whitehill R, et al: The nonsurgical management of odontoid fractures in adults. Spine 9:229-230, 1984

42. White AA III, Panjabi MM: Clinical Biomechanics of the Spine, ed 2. Philadelphia: JB Lippincott, 1990, pp 610-611

Manuscript received April 15, 2000.

Accepted in final form May 25, 2000.

Address reprint requests to: Ronald I. Apfelbaum, M.D., Department of Neurosurgery, University of Utah Health Sciences Center, 50 North Medical Drive, Salt Lake City, Utah 84132. email: ronald.apfelbaum@hsc.utah.edu.

This article is a preview article for the Journal of Neurosurgery: Spine, October 2000. 